\title{
Determination of wastewater color by integral spectrophotometry based on complementary color
}

\author{
Jinlei Bao ${ }^{1,2, a}$, Tao Li ${ }^{1, b}$, Baozeng Ren ${ }^{1, c}$ \\ ${ }^{1}$ School of Chemical Engineering and Energy, Zhengzhou University, Zhengzhou, China \\ ${ }^{2}$ School of Resources and Environment, Henan University of Engineering, Zhengzhou, China \\ abao_jinlei@126.com, blitao_0912@126.com, crenbz@zzu.edu.cn
}

\begin{abstract}
Keywords: wastewater color; color determination; complementary color; spectral curve area Abstract. The standard method to determine industrial effluents color is multiple dilution method which has defects of reproducibility, obscure endpoint of dilution, and subjective influence. For this, based on the theory of complementary color, an integral spectrophotometry method was proposed to determine the color of industrial effluents. The results from vary hues show that there is a good linear relationship between the spectral curve area $\mathrm{S}$ and the color $\mathrm{C}$ in the selected wavelength range, and all the correlation coefficient $\mathrm{R}^{2}$ are more than 0.99 . The method validation by pharmaceutical wastewater shows that the method is feasible and its results are consistent with that of the dilution method. All the relative error are less than $5 \%$, and the method have merits of sound stability and reproducibility. So, it can be widely used as a substitute for multiple dilution method to determine the color of industrial wastewaters.
\end{abstract}

\section{Introduction}

The color of water and wastewater is not only an aesthetic issue but also an important water quality parameters, it effects the photosynthesis of water, causes dissolved oxygen loss, in turn, leads to ecological imbalance[1], in addition, some colored substances may be toxic or carcinogenic[2]. Therefore, color is an important control parameter of wastewater discharge standards. China's current standard methods to determine color of water and wastewater are platinum-cobalt method and multiple dilution method, the former applies to natural water and the latter is suitable for industrial wastewater, moreover, the results of the two methods are not comparable [3]. The platinum-cobalt method requires comparison of the samples and the standard solutions or color charts by a person, and the multiple dilution method requires to determine the dilution end point by naked eyes. For platinum-cobalt method, when the sample color is 5-25 TCU (True Color Unit), the difference is not obvious and the error is larger[4]. For multiple dilution method, due to the ability to perceive color varies, and environmental factors also affect people's perception of color, so it is subjective to judge the end point of dilution, thus the results have strong subjectivity and uncertainty, which limits the application of the standard method. In order to overcome these drawbacks, spectrophotometers are used to replace the human eyes to judge the dilution endpoint or to compare with the standard solution. These methods are fixed wavelength method, AMDI3, 31 method, and area integral method [5]. For fixed wavelength method, the maximum absorption wavelength, max, or other suitable characteristic wavelength is found by spectral scanning, and then the relationship between color and absorbance is established. The working wavelength of this method is mostly in the ultraviolet region, which is inconsistent with the human eye's perception of color. According to the principle of color rendering, the human eye only responds to visible light [6], and the absorption characteristics of the object in the ultraviolet region should be independent of its color. In addition, it is dubious to use standard curve determined by platinum cobalt to measure the color of water samples, in which the composition may varies badly from the color scales. According to the AMDI method, the color of water is measured by 3 or 31 wavelengths which uniformly selected in the visible range $(400-760 \mathrm{~nm})$ [7]. The ADMI method is accurate and reliable, and has been adopted as a standard method for color determination of wastewater in many countries [8]. However, the principle of this method is different from that of human perception of color, moreover, all China's discharge standards of wastewaters are based on the 
multiple dilution method, and so there is a problem to transform the results to that of standard method (GB11903-89). Area method (integral method) was proposed to measure wastewater color by replacement of fixed wavelength with that of whole visible region [9]. However, the workload of this method is very large and not all consistent with the human eye's perception of color.

Based on the complementary theory, a material absorbs certain wavelength light in the visible light, and it shows the complementary color, therefore, the color human perceived should be a range of visible light, rather than a single or a few fixed wavelengths, nor the whole visible spectrum [10].Therefore, the spectral range of absorption can be determined according to the color of water, and the color can be determined only by measuring the absorbance in this region. Based on the theory of complementary color, the color of wastewater is measured by integral spectrophotometry. In which color scales (potassium hexachloroplatinate and cobalt chloride) are not required. The results are consistent with that of the national standard (dilution multiple method).

\section{Materials and method}

\section{Experimental principle}

The color of matter is determined by its selective absorption of light at different wavelengths. If a substance selectively absorbs a certain light, the light transmitted is its complementary light, which is the color of the substance. The relationship between the color of a substance and absorbed light wavelength is shown in Table 1[10]. Thus the perception of a substance's color is not a fixed wavelength, but a wavelength range. According to the color of a substance, the wavelength range of the absorbed light can be determined, and the absorption spectrum area (S) in the selected wavelength range can be determined, finally the relationship between $\mathrm{S}$ and the color(C) is determined.

Table 1 relationship between wavelength of absorbed light and hue

\begin{tabular}{ccc}
\hline $\begin{array}{c}\text { Absorbed } \\
\text { wavelength(nm) }\end{array}$ & $\begin{array}{c}\text { Hue of } \\
\text { absorbed } \\
\text { light }\end{array}$ & Perceived hue \\
\hline $400-440$ & Violet & Greenish-yellow \\
$440-480$ & Blue & Yellow \\
$480-510$ & Blue-green & Orange \\
$510-540$ & Green & Red \\
$540-570$ & Yellow-green & Purple \\
$570-580$ & Yellow & Blue \\
$580-610$ & Orange & Greenish-blue \\
$610-700$ & Red & Blue-green \\
\hline
\end{tabular}

\section{Reagents and apparatus}

According to the human eye's sensitivity to different hues, red, green, blue and purple solution were chosen as research object. Each hue was made from different dyes, red: rose-Bengal, eosin-Y, and red ink, green: malachite green, bromocresol green, blue: methylene blue, cupric sulfate, blue ink, violet: potassium permanganate, crystal violet. The absorbance of the solution was determined by a UV-Vis spectrophotometer (UV-2700). In addition to red ink, blue ink for the commercial, the remaining reagents are analytical pure.

\section{Experimental procedures}

(1) According to different hues, certain concentration of colored solutions were prepared with dyes.

(2) Dilute the solution with distilled water to colorless, then the dilution factor is $\mathrm{n}$. Assuming the original concentration is 1 , then the relative concentration of the diluted solution is to $1 / \mathrm{n}$. 
(3) According to the color of the solution and table.1 to determine the absorption wavelength range, in which spectral curve was determined by a spectrophotometer, and then the area of the curve(S) was calculated.

(4) Define the relationship between spectral curve area(S) and dilution factor $n$.

(5) Method validation

a. Determine the working curve: Taken a pharmaceutical wastewater as example, whose color was yellow-brown, and its color (C) was determined by multiple dilution method [3], meanwhile spectral curve was measured, and curve area(S) was calculated, corresponding to the dilution factors. Finally C-S working curve was got.

b. Method validation: Taken other pharmaceutical wastewater sample, its spectral curve area (S)were measured by method mentioned above, then its color was calculated by the working curve, and compared with that determined by standard method (multiple dilution method), then the method was validated.

\section{Results and discussions}

\section{Relationship between spectral curve $\operatorname{area}(S)$ and dilution factor $n$.}

In the wavelength range selected on complementary color theory, the relationship between the spectral area (S) of different hue solutions and the dilution factors (n) was shown in Figure 1, a (green hues), b(red hues),c(violet hues),d(blue hues). The Figure1 shows that, in an appropriate concentration range, there is a sound linear relationship between spectra area $\mathrm{S}$ and dilution factor $\mathrm{n}$. all the correlation coefficient $\mathrm{R}^{2}$ are greater than 0.99 , it is indicated that the relationship between the color and spectral curve area of samples can be quantitatively calculated. Therefore, it is only necessary to measure the color an industrial wastewater according to the multiple dilution method, and establish the linear relationship between the color of wastewater and spectra curve area S, and then it can be used to determine the color of specific wastewater.
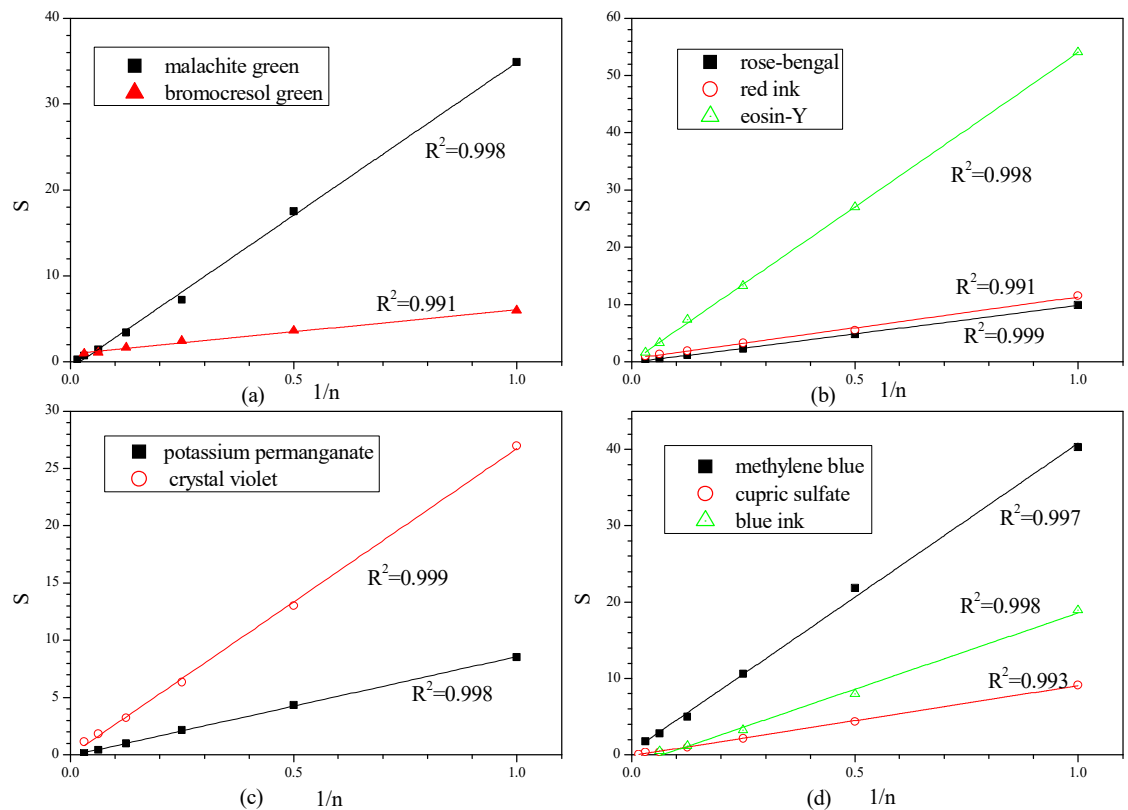

(d) $1 / \mathrm{n}$

Figure 1 Relationship of spectra curve area and dilution factor of different color aqueous solutions

\section{Method validation}

A case study of a pharmaceutical effluents was conducted to verify the feasibility of this method, first its color $\mathrm{C}$ was determined by multiple dilution method, and then absorption wavelength range 
was found in Table 1 according to its appear color, finally, the C-S working curve showed in Figure 2 was worked out by the method mentioned above. At different time, the color of pharmaceutical effluents was measured by multiple dilution method of dilution and this method. The results were shown in Table 2. The results show that this method is consistent with the dilution multiple method, their results have same accuracy. Moreover, thanks to its sound stability, for a particular wastewater, once the C-S working curve has been worked out, its color can be determined quickly, and the results will not vary from person to person, therefore, to overcome the influence of subjective factors.

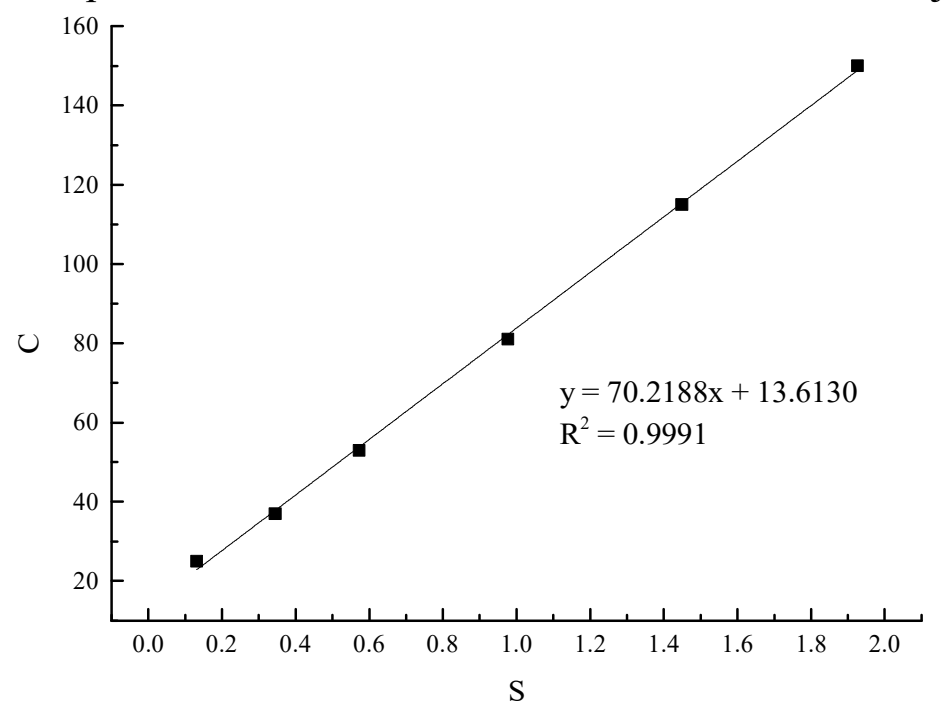

Figure 2 color working curve of the pharmaceutical effluents

Table 2 color determined by method of multiple dilution and this article

\begin{tabular}{ccccccc}
\hline method & Sample 1 & Sample 2 & Sample 3 & Sample 4 & Sample 5 & Sample 6 \\
\hline Multiple method & 27.5 & 47.5 & 50 & 84 & 88 & 135 \\
The method of this article & 26.79 & 48.58 & 47.61 & 81.40 & 90.46 & 131.70 \\
RSD\% & 2.58 & 2.27 & 4.78 & 3.09 & 2.79 & 2.44 \\
\hline
\end{tabular}

\section{Conclusions}

This method does not require the expensive color standard solution, and its results are consistent with the multiple dilution method. For a particular industrial wastewater, once the C-S working curve has been worked out by a professional operator, it can be used for colorimetric determination of similar wastewater. Moreover, the results are reproducible, and the subjective effects of different personnel on are overcome, and the results are comparable. So, it can be widely used to determine the color of industrial wastewaters.

\section{References}

[1] Kumar P, Kumar S, Bhardwaj N K. Color Removal from Kraft Pulp Bleaching Wastewater Using Heterogeneous Photocatalysis[J]. Indian Pulp and Paper Technical Association Journal, 2011, 23(1).

[2] Kao C M, Chou M S, Fang W L, et al. Regulating colored textile wastewater by $3 / 31$ wavelength ADMI methods in Taiwan[J]. 2001, 44(5):1055-1063.

[3] Kumar S. APHA, AWWA and WEF Standard Methods for the Examination of Water and Wastewater, 20th edition, American Public Health Association, American Water Works Association and Water Environment Federation, Washington, DC, 1998.[M]. 2013. 
[4] Chen $\mathrm{H}$, Chen $\mathrm{Y}$, Zhan $\mathrm{H}$, et al. Determination of chroma in pulping effluent by ratio spectrum-derivative spectrophotometry[J]. Environmental Progress \& Sustainable Energy, 2010, 29(3):342-348.

[5] Hao O J, Kim H, Chiang P. Decolonization of Wastewater [J]. Critical Reviews in Environmental Science \& Technology, 2000, 30(4):449-505.

[6] J.R, D. T, X. C.Technology: instrumental and operational impacts on Spectrophotometer color Measurements [J]. The Journal of Cotton Science, 2008, 12(3).

[7] Hongve D, Åkesson G. Spectrophotometric determination of water colour in hazen units [J]. Water Research, 1996, 30(11):2771-2775.

[8] Yu R F, Chen H W, Cheng W P, et al. Measurements of wastewater true color by 4/6 wavelength methods and artificial neural network[J]. Environ Monit Assess, 2006, 118(1-3):195-209.

[9] Wu J, Eiteman M, Law S. Evaluation of Membrane Filtration and Ozonation Processes for Treatment of Reactive-Dye Wastewater [J]. Journal of Environmental Engineering, 1998, 124(3):272-277.

[10] Ingamells W. Colour for textiles: a user's handbook [M]. Society of Dyers and Colourists, 1993. 